\title{
In memoriam: Ludwig G. Strauss, MD, 1949-2013
}

\author{
Antonia Dimitrakopoulou-Strauss • Ignasi Carrió
}

Published online: 7 June 2013

(C) Springer-Verlag Berlin Heidelberg 2013

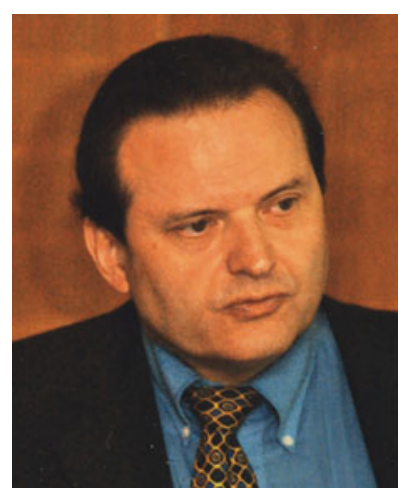

Ludwig G. Strauss, MD, a nuclear medicine physician and radiologist who pioneered the field of nuclear oncology, died on 29 May 2013 at his home in the German state of Hessen after a long fight against his cancer disease. Strauss was born in 1949 in Worms and grew up nearby. He studied medicine and mathematics in parallel at the Justus-Liebig University of Giessen. He completed his studies in 1976 and received his medical degree in 1978 after submitting a doctoral thesis. From 1975 to 1978 he worked as a resident for internal medicine. His interest was focussed on the field of nuclear

\footnotetext{
A. Dimitrakopoulou-Strauss $(\square)$

Clinical Cooperation Unit Nuclear Medicine, E 060, German Cancer Research Center, Im Neuenheimer Feld 280, 69120 Heidelberg, Germany

e-mail: ads@ads-lgs.de

A. Dimitrakopoulou-Strauss

e-mail: a.dimitrakopoulou-strauss@dkfz.de

I. Carrió

Nuclear Medicine Department, Autonomous University of Barcelona, Hospital Sant Pau, Sant Quintí 89, 08025 Barcelona, Spain
}

medicine from an early stage. In 1978 he moved to the German Cancer Research Center in Heidelberg and began his career as research fellow in the Department of Nuclear Medicine. In 1982 he completed his residency in nuclear medicine and decided to continue on the field on radiology. He was resident in radiology at the University Clinics of Mannheim from 1982 to 1985, and he finalized his specialization in radiology in 1986. He then served as a supervising radiologist, and become an assistant professor in radiology in 1986. His special interest at that time was the application of computed tomography and single photon emission tomography in cancer diagnostics. In 1987 he again transferred to the German Cancer Research Center and worked as a Director of the Medical-PET and Biological Imaging Group. In 1992 he become a full professor of radiology, a position he held until his death. Strauss introduced positron emission tomography (PET) to nuclear oncology and pioneered the field of tumor diagnostics. His research team was the first to use radiolabeled cytostatic drugs, such as F-18-fluorouracil to monitor the therapeutic effect in colorectal cancer. Furthermore, he introduced F-18-FDG for tumor diagnostics and therapy monitoring. This work was published in the Journal of Nuclear Medicine in 1991 under the title "The applications of PET in clinical oncology." This ground-breaking paper, written with Peter S. Conti, has been cited over 800 times to date. This is his bestknown work, and the results of these studies are widely acknowledged to have influenced new research in this field.

His group studied different tracers for tumor diagnostics, such as $\mathrm{O}-15$-water for perfusion assessment, N-13-glutamate and C-11-aminoisobutyric acid (AIB) for amino acid transport, F-18-DOPA, F-18-FLT for proliferation, F-18-fluoride, F-18-MISO for hypoxia, and receptor-active tracers such as Ga-68-DOTATOC and Ga-68-bombesin. The individualization of cancer therapy was another major research interest. He proposed the use of quantification not only based on SUV but 
also using compartmental and non-compartmental methods, and did significant work to optimize the software for that purpose. Parametric imaging was another important research field.

Strauss trained radiologists and nuclear medicine physicians as well as guest scientists from many different countries during his career. He served as a reviewer for several high-impact-factor journals as well as for congresses and grant applications.

Ludwig G. Strauss was much more than a scientist. He was a pilot, a technology enthusiast, a world traveller, a piano player, and a passionate optimist. He is survived by his wife, his three children, and his legacy in nuclear medicine. 